\title{
Time-dependent resonant tunneling via two discrete states
}

\author{
T. H. Stoof and Yu. V. Nazarov \\ Department of Applied Physics and Delft Institute for Microelectronics and Submicrontechnology, Delft University of Technology, \\ Lorentzweg 1, 2628 CJ Delft, The Netherlands
}

(Received 7 June 1995; revised manuscript received 28 September 1995)

\begin{abstract}
We theoretically investigate time-dependent resonant tunneling via two discrete states in an experimentally relevant setup. Our results show that the dc transport through the system can be controlled by applying external irradiation with a frequency which matches the energy difference between the discrete states. We predict resonant phenomena which should be easily observable in experiments.
\end{abstract}

Time-dependent tunneling phenomena have received increasing attention in recent years. In the early eighties, Büttiker and Landauer studied the tunneling time needed for an electron to traverse a potential barrier. ${ }^{1}$ More recent theoretical work focused on the time-dependence of resonant tunneling using an effective Schrödinger equation ${ }^{2}$ and on a description of the time-dependent current through mesoscopic structures in terms of nonequilibrium Green's functions. ${ }^{3}$ In addition, the considerable improvement in nanofabrication techniques facilitated some interesting experimental studies. Kouwenhoven et al. measured the photon-assisted tunneling current through a single quantum dot with an effectively continuous level spectrum, due to thermal smearing. ${ }^{4}$ van der Vaart et al. studied the dc current through a double dot system, with well developed OD states in each dot and clearly resolved resonances between energy levels in both dots. ${ }^{5}$ The sharp resonance features make it very tempting to perform experiments with time-dependent fields. The dc current through such a structure in the presence of oscillating fields may be expected to display interesting phenomena, not observable in a single dot.

Some time-dependent aspects of resonant tunneling via two wells in layered semiconductor heterostuctures have been studied in Refs. 6,7. However, the states in such stuctures are not really discrete and it is plausible to disregard Coulomb blockade effects. This makes it impossible to apply the results of these works to realistic ultrasmall quantum dots.

In this paper, we use the density matrix approach of Ref. 8 , in which the resonant states, being true quantummechanical many-body states of the two dots, are described by a time-dependent tunneling Hamiltonian. Transitions between nonresonant states of the system are taken into account through a master equation for the density matrix elements. We calculate the photoresponse of the system in several experimentally relevant limits and derive an explicit expression for the shape of the resonant peaks in the case of an external perturbation with arbitrary amplitude. Close to resonance, the dc current is found to be very sensitive to the oscillating field. The satellite resonances induced by the external oscillating field can be of the same order of magnitude as the main static resonance with an even smaller width.

The system under consideration (Fig. 1) consists of two quantum dots $A$ and $B$ in series. The dots are connected by tunnel junctions to two large reservoirs $L$ and $R$, which are assumed to have continuous energy level spectra and are filled up to their respective Fermi energies. If we neglect all tunneling processes, a system of discrete many-body states is formed in each dot. The best conditions for transport occur when it costs no energy to transfer an electron between the dots, i.e., the energy difference between a state with one extra electron in the left dot and a state with one extra electron in the right dot is zero. In the experiment in Ref. 5, this energy difference could be tuned by an external gate voltage. The current through the system vs gate voltage consists of a series of peaks corresponding to the resonances between different discrete states. There could be a variety of different transport processes occuring in a resonance point, as described in Ref. 8. We concentrate on the simplest experimentally relevant case, namely, when the resonance occurs between the ground states of both dots. We assume that the bias voltage is much larger than the temperature and the energy difference between the states in resonance. Consequently, electrons can only enter the two-dot system from the left and leave it only to the right. Transitions from the left and to the right lead are possible with rates $\Gamma_{L}$ and $\Gamma_{R}$, respectively. Here and throughout the paper, units are used such that $\hbar=1$. Under the conditions mentioned above, the tunnel rates close to a resonance point will depend weakly on the bias voltage and we will, therefore, treat them as constants. We will assume that the voltage applied is smaller than the Coulomb threshold for adding yet another electron so that it

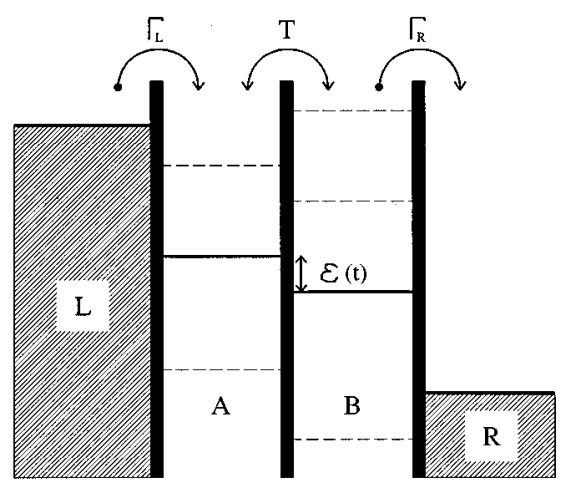

FIG. 1. Schematic picture of the system. Two quantum dots $A$ and $B$ are coupled to leads $L$ and $R$ via tunnel junctions. Transitions are possible with rates $\Gamma_{L}$ and $\Gamma_{R}$. The tunneling rate between the dots is $T$ and the energy difference between the levels is denoted by $\epsilon(t)$. 
is impossible, due to the Coulomb blockade, for an electron to tunnel into the system, while another electron is still present in either one of the dots. We concentrate on two states only and disregard other states, which is allowed in the neighborhood of the resonance. The system can also be in a third state $|0\rangle,{ }^{9}$ when there is no extra electron in either one of the dots. The energies of the resonant states, which both lie well between the electrochemical potentials in the left and right lead, initially differ by an amount $\epsilon_{0}$. Under these conditions the transport through the system depends only very weakly on the bias voltage, but does depend strongly on the gate electrode via the energy difference $\epsilon_{0}$. We assume that a time-dependent oscillating signal is applied to the gate electrode, so that the time-dependent energy difference becomes $\epsilon(t)=\epsilon_{0}+\tilde{\epsilon} \cos \omega t$, where $\tilde{\epsilon}$ is the amplitude and $\omega$ the frequency of the externally applied signal.

The dynamics of the resonant states, $|a\rangle$ and $|b\rangle$, is governed by the time-dependent tunneling Hamiltonian $\mathscr{H}(t)=\mathscr{H}_{0}(t)+\mathscr{H}_{T}$, where $\mathscr{H}_{0}$ is given by

$$
\mathscr{H}_{0}(t)=\frac{1}{2} \epsilon(t)(|a\rangle\langle a|-| b\rangle\langle b|)
$$

and $\mathscr{H}_{T}$ describes the coupling between the dots that introduces mixing between the eigenstates $|a\rangle$ and $|b\rangle$ of the system:

$$
\mathscr{H}_{T}=T(|a\rangle\langle b|+| b\rangle\langle a|) .
$$

The average current through the system is given by

$$
\langle I\rangle / e=\operatorname{Tr}(\rho \mathscr{T}),
$$

where $\mathscr{T}$ is the current operator:

$$
\mathscr{T}=i T(|a\rangle\langle b|-| b\rangle\langle a|),
$$

and $\rho$ is the density matrix for the two-level system. We describe transitions between different states in the density matrix approach. ${ }^{8}$ The equations for the density matrix elements read

$$
\begin{gathered}
\partial \rho_{a} / \partial t=+\Gamma_{L} \rho_{0}+i T\left(\rho_{b a}-\rho_{a b}\right), \\
\partial \rho_{b} / \partial t=-\Gamma_{R} \rho_{b}-i T\left(\rho_{b a}-\rho_{a b}\right), \\
\partial \rho_{a b} / \partial t=-\frac{1}{2} \Gamma_{R} \rho_{a b}+i \epsilon(t) \rho_{a b}+i T\left(\rho_{b}-\rho_{a}\right), \\
\partial \rho_{b a} / \partial t=-\frac{1}{2} \Gamma_{R} \rho_{b a}-i \epsilon(t) \rho_{b a}-i T\left(\rho_{b}-\rho_{a}\right),
\end{gathered}
$$

where $\rho_{a}, \rho_{b}$ and $\rho_{0}=1-\rho_{a}-\rho_{b}$ denote the probabilities for an electron to be in the left dot, the right dot, or in neither dot, respectively, and $\rho_{a b}=\rho_{b a}^{*}$ are the nondiagonal density matrix elements. In these equations, the terms proportional to $\Gamma_{L}$ and $\Gamma_{R}$ describe the transitions to and from the reservoirs between the states $|0\rangle$ and $|a\rangle$ and the states $|b\rangle$ and $|0\rangle$, respectively. All other terms follow from the Liouville equation: $i d \rho / d t=[\mathscr{H}, \rho]$. Note that the rates $\Gamma_{L}$ and $\Gamma_{R}$ do not enter the equations in a symmetric way. $\Gamma_{R}$ describes the decay of the resonant states, whereas $\Gamma_{L}$ describes the buildup of these resonant states.

The relevant energy scales of the system are the transition rates $\Gamma_{L}$ and $\Gamma_{R}$, the tunneling amplitude $T$, and the frequency $\omega$ and amplitude $\tilde{\epsilon}$ of the applied perturbation. There are three limiting cases for which we can develop an analytical approach to the problem. They are complementary and essentially cover all the interesting physics.

We will first consider the limiting case of a small perturbation amplitude; $\tilde{\epsilon} \ll \omega, T, \Gamma_{L, R}$. Using the fact that $\rho_{0}=1-\rho_{a}-\rho_{b}$, we rewrite Eq. (5) in matrix notation:

$$
\partial \vec{\rho} / \partial t=\left(\hat{\Gamma}+\hat{T}+\hat{\epsilon}_{0}+\hat{\epsilon} \cos \omega t\right) \vec{\rho}+\vec{c}
$$

where $\vec{\rho}=\left(\rho_{a}, \rho_{b}, \rho_{a b}, \rho_{b a}\right)^{T}, \vec{c}=\left(\Gamma_{L}, 0,0,0\right)^{T}$, and the matrices $\hat{\Gamma}, \hat{T}, \hat{\epsilon}_{0}$, and $\hat{\boldsymbol{\epsilon}}$ correspond to Eq. (5). The stationary solution of these equations without irradiation is

$$
\vec{\rho}_{0}=-\left(\hat{\Gamma}+\hat{T}+\hat{\epsilon}_{0}\right)^{-1} \vec{c} .
$$

This determines the shape of the stationary resonant peaks observed by van der Vaart et al.:

$$
I_{\text {stat }}=T^{2} \Gamma_{R} /\left[T^{2}\left(2+\Gamma_{R} / \Gamma_{L}\right)+\Gamma_{R}^{2} / 4+\epsilon_{0}^{2}\right] .
$$

The first order correction to the stationary solution is

$$
\vec{\rho}_{1}=\vec{\rho}_{1}^{+} \exp (i \omega t)+\vec{\rho}_{1}^{-} \exp (-i \omega t)
$$

with $\vec{\rho}_{1}^{ \pm}$the positive and negative frequency part, respectively:

$$
\vec{\rho}_{1}^{ \pm}=-\left(\hat{\Gamma}+\hat{T}+\hat{\epsilon}_{0} \mp i \omega \hat{I}\right)^{-1}(\hat{\epsilon} / 2) \vec{\rho}_{0},
$$

$\hat{I}$ being the unit matrix. This contribution contains only oscillatory terms, which average out when calculating the dc current. We, therefore, also determine the second order correction terms (proportional to $\tilde{\epsilon}^{2}$ ) and obtain

$$
\vec{\rho}_{2}=-\left(\hat{\Gamma}+\hat{T}+\hat{\epsilon}_{0}\right)^{-1}(\hat{\epsilon} / 2)\left(\vec{\rho}_{1}^{+}+\vec{\rho}_{1}^{-}\right)
$$

Using the density matrix elements $\vec{\rho}_{2}$ we may calculate the photoresponse of the system. This quantity can be easily measured experimentally by slowly modulating the irradiation amplitude. ${ }^{4}$ In Fig. 2, a plot is given of the photoresponse as a function of $\epsilon_{0}$ and $\omega$ for $\Gamma_{L}=\Gamma_{R}=0.2 T$. The figure clearly shows resonant satellite peaks for $\omega$ and $\epsilon_{0}$ satisfying $\omega^{2}=\epsilon_{0}^{2}+4 T^{2}$, i.e., resonant modes occur when the frequency of the applied perturbation matches the renormalized energy difference $\sqrt{\epsilon_{0}^{2}+4 T^{2}}$ of the two levels. For frequencies below $2 T$, there are no satellite peaks, because the energy $\hbar \omega$ of the photon is smaller than the energy level spacing. The evolution of a resonant satellite peak is shown in Fig. 3, where a current peak for $\omega=3 T$ has been plotted vs $\epsilon_{0}$ for different values of $\Gamma_{R} / T=\Gamma_{L} / T$. We see that the peak can be seen even at moderately large values of $\Gamma_{R} / T$, but the best resonance conditions occur when $\Gamma_{R} \ll \max \left(T, \epsilon_{0}\right)$ and $\omega=\sqrt{\epsilon_{0}^{2}+4 T^{2}}$.

We have developed a second approach, which allows us to explore the satellite peak at arbitrary values of irradiation amplitude $\tilde{\boldsymbol{\epsilon}}$ under the conditions mentioned above. Substituting $\vec{\rho}=\vec{\rho}_{0}+\vec{\rho}_{+}(t) \exp (i \omega t)+\vec{\rho}_{-}(t) \exp (-i \omega t)$ in Eq. (6) and neglecting terms proportional to $\exp ( \pm 2 i \omega t)$, we obtain

$$
\begin{array}{r}
\partial \vec{\rho}_{0} / \partial t=\hat{\Gamma} \vec{\rho}_{0}+\left(\hat{\epsilon}_{0}+\hat{T}\right) \vec{\rho}_{0}+(\hat{\epsilon} / 2)\left(\vec{\rho}_{+}+\vec{\rho}_{-}\right)+\vec{c} \\
\partial \vec{\rho}_{+} / \partial t=\hat{\Gamma} \vec{\rho}_{0}+\left(\hat{\epsilon}_{0}+\hat{T}-i \omega \hat{I}\right) \vec{\rho}_{+}+(\hat{\epsilon} / 2) \vec{\rho}_{0} \\
\partial \vec{\rho}_{-} / \partial t=\hat{\Gamma} \vec{\rho}_{-}+\left(\hat{\epsilon}_{0}+\hat{T}+i \omega \hat{I}\right) \vec{\rho}_{-}+(\hat{\epsilon} / 2) \vec{\rho}_{0}
\end{array}
$$

Near the resonance point, we can approximate the solution $\vec{\rho}$ by an expansion in terms of the eigenvectors of the matrix $\hat{\epsilon}_{0}+\hat{T}: \quad \vec{\rho}_{0}=\alpha_{1} \vec{v}_{1}+\alpha_{2} \vec{v}_{2}, \quad \vec{\rho}_{+}=\alpha_{+} \vec{v}_{+}, \quad$ and $\vec{\rho}_{-}=\alpha_{-} \vec{v}_{-}$, where $\vec{v}_{1}$ and $\vec{v}_{2}$ are the eigenvectors with eigenvalue 0 and $\vec{v}_{ \pm}$those with eigenvalues $\pm i \sqrt{\epsilon_{0}^{2}+4 T^{2}}$. We obtain a set of four closed equations for the coefficients $\alpha_{1,2,+,-}$ by taking the inner product of Eq. (12a) with $\vec{v}_{1}$ and $\vec{v}_{2}$, of Eq. (12b) 


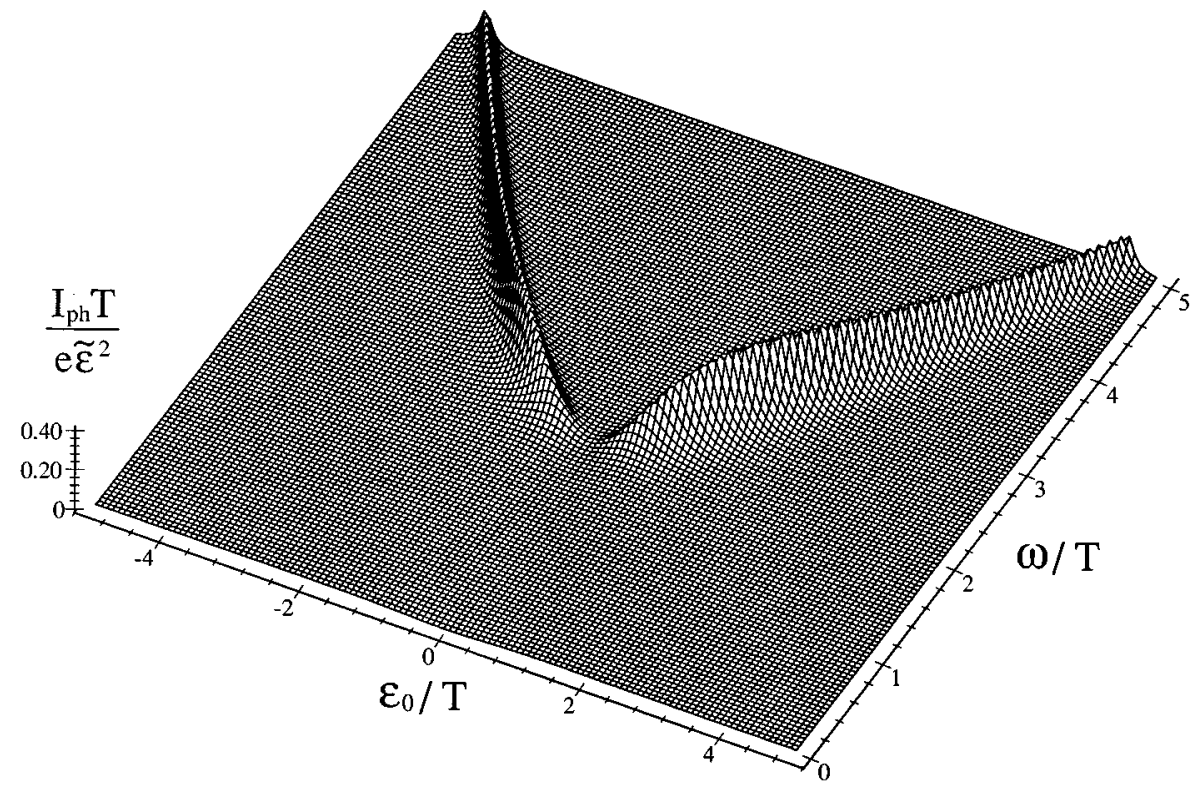

FIG. 2. Scaled photoresponse of the system, as a function of the energy difference $\epsilon_{0} / T$ between the levels and the frequency $\omega / T$ of the applied signal. The plot was made with $\Gamma_{L}=\Gamma_{R}=0.2 T$.

with $\vec{v}_{+}$, and of Eq. (12c) with $\vec{v}_{-}$. Solving for the stationary solution and calculating the current profile near the resonance point $\epsilon_{r}=\sqrt{\omega^{2}-4 T^{2}}$ results in a Lorentzian line shape:

$$
I / e=I_{\max } w^{2} /\left[w^{2}+\left(\epsilon_{0}-\epsilon_{r}\right)^{2}\right],
$$

with height

$$
I_{\max }=\tilde{\epsilon}^{2} \Gamma_{R}\left(\alpha^{2}-4\right) / \gamma\left(\gamma \Gamma_{R}^{2}+\beta \tilde{\epsilon}^{2}\right),
$$

and half width at half maximum

$$
w=\left(\alpha / 2 \sqrt{\alpha^{2}-4}\right) \sqrt{\Gamma_{R}^{2}+\beta / \gamma \tilde{\epsilon}^{2}},
$$

where $\alpha=\omega / T, \beta=\Gamma_{R} / \Gamma_{L}+2$, and $\gamma=\alpha^{2}+\beta-4$.

In the limit of small amplitude, the height of the current peak scales with the square of $\tilde{\epsilon}, I_{\max }=\tilde{\epsilon}^{2}\left(\alpha^{2}-4\right) / \gamma^{2} \Gamma_{R}$, whereas the width remains constant: $w=\frac{1}{2} \alpha \Gamma_{R} / \sqrt{\alpha^{2}-4}$ $\sim \Gamma_{R}$, consistent with the results presented in Fig. 3. At $\alpha=2$ (corresponding to $\epsilon_{0}=0$ ) the peak vanishes, as seen in Fig. 2. With further increase of $\tilde{\boldsymbol{\epsilon}}$ the current saturates at a value of $I_{\text {sat }}=\Gamma_{R}\left(\alpha^{2}-4\right) / \beta \gamma$, which is of the order of the

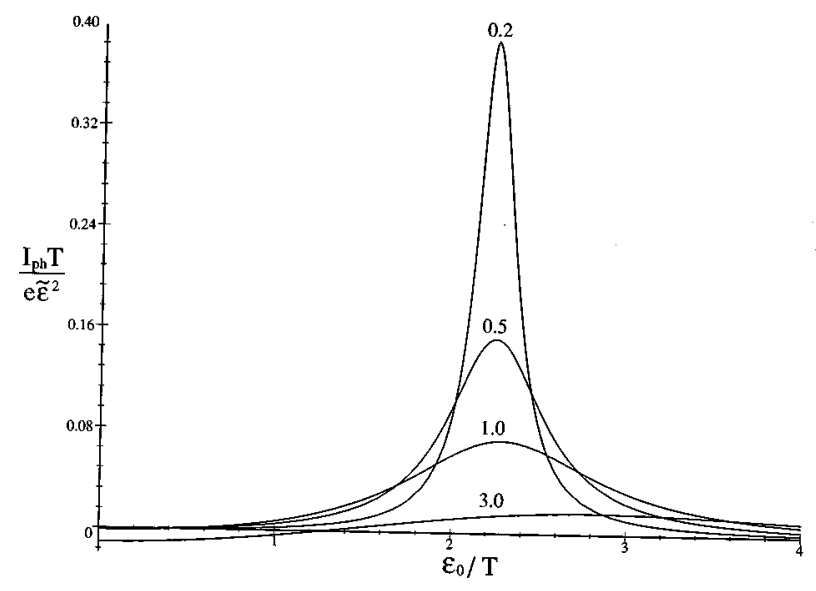

FIG. 3. Evolution of a satellite peak for ratios $\Gamma_{L} / T=\Gamma_{R} / T=0.2,0.5$, 1.0, and 3.0, respectively. The plots were made for a frequency of $\omega=3 T$. height of the stationary peak. This saturation occurs at relatively small $\tilde{\boldsymbol{\epsilon}} \sim \Gamma_{R}$. The width of the peak increases with growing $\tilde{\epsilon}$. At $\tilde{\epsilon} \gg \Gamma_{R}$, it is proportional to $\tilde{\epsilon}$.

Therefore we have shown that, under good resonance conditions, the current is very sensitive to the external irradiation. A relatively weak irradiation induces a big satellite peak that has a much smaller width than the stationary one.

For small tunneling amplitudes $T$, provided $\epsilon_{0} \gg \Gamma_{R}$, the height scales with $T^{2}: I_{\max }=T^{2} \tilde{\epsilon}^{2} / \Gamma_{R} \omega^{2}$, and the half width reduces to $w=\frac{1}{2} \Gamma_{R}$. These results agree with the expression for the photon-assisted tunneling current derived below, where we consider our third approach, in which the tunneling amplitude is small compared to all other energy scales in the system; $T \ll \tilde{\epsilon}, \omega, \Gamma_{L, R}$.

First, we perform a transformation on the density matrix that leaves the diagonal elements invariant and which changes the nondiagonal elements as follows:

$$
\bar{\rho}_{a b}=\rho_{a b} \exp \left(-i \int_{-\infty}^{t} d \tau \epsilon(\tau)\right),
$$

This transformation eliminates the explicit time dependence in Eqs. (5c) and (5d) and introduces it in the transformed tunneling amplitude. The equations for the nondiagonal density matrix elements now assume the form

$$
\partial \bar{\rho}_{a b} / \partial t=\left(i \epsilon_{0}-\frac{1}{2} \Gamma_{R}\right) \bar{\rho}_{a b}+i \bar{T}(t)\left(\bar{\rho}_{b}-\bar{\rho}_{a}\right),
$$

with the time-dependent tunneling amplitude,

$$
\bar{T}(t)=T \exp \left(i \int_{-\infty}^{t} d \tau \epsilon(\tau)\right) .
$$

The equations for $\bar{\rho}_{b a}$ are simply the complex conjugate of Eqs. (16), (17), and (18). For $\bar{\rho}_{a b, \omega}$ in the lowest nonvanishing order in $T$, we obtain

$$
\bar{\rho}_{a b, \omega}=i \bar{T}_{\omega} /\left[\frac{1}{2} \Gamma_{R}+i\left(\omega-\epsilon_{0}\right] .\right.
$$

Expanding $\bar{T}(t)$ in a Fourier series;

$$
\bar{T}(t)=T \sum_{n=-\infty}^{\infty} J_{n}(\tilde{\epsilon} / \omega) \exp (-i n \omega t),
$$




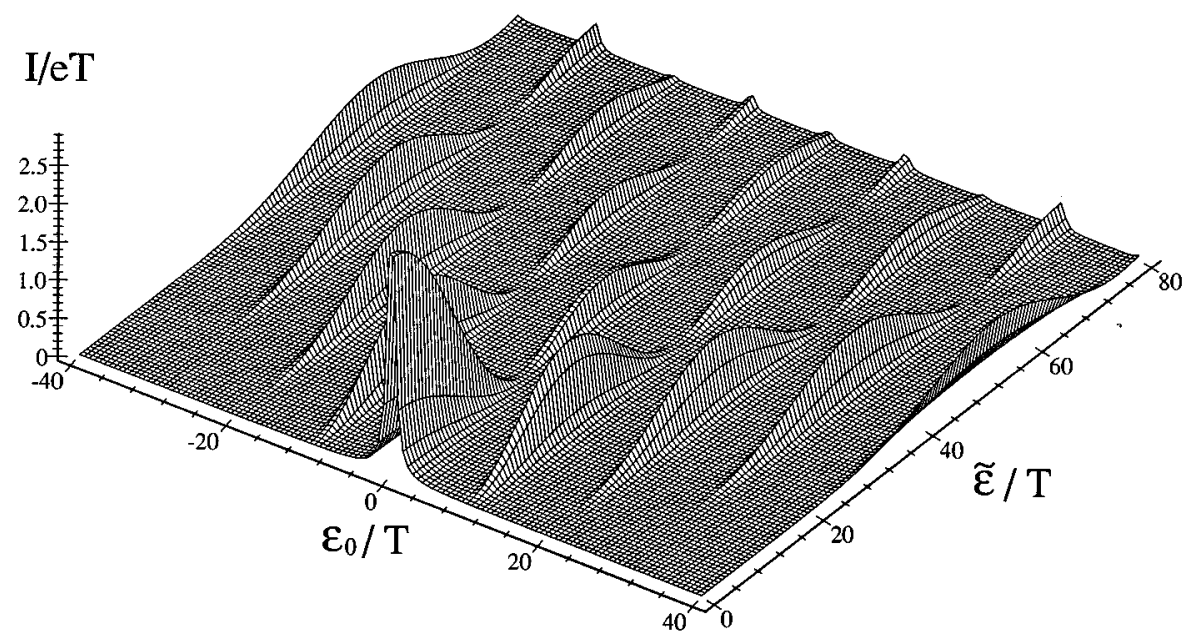

FIG. 4. Scaled current through the dots, as a function of the energy difference $\epsilon_{0} / T$ between the levels and the amplitude $\tilde{\epsilon} / T$ of the applied signal. The plot was made with $\Gamma_{R}=T$ and $\omega=10 T$.

and calculating the dc current, Eq. (3), results in

$$
\langle I\rangle / e=T^{2} \Gamma_{R} \sum_{n=-\infty}^{\infty} J_{n}^{2}(\tilde{\epsilon} / \omega) /\left[\frac{1}{4} \Gamma_{R}^{2}+\left(n \omega-\epsilon_{0}\right)^{2}\right],
$$

where $J_{n}$ is a $n$th order Bessel function of the first kind. This equation for the current is similar to the expression found by Tien and Gordon ${ }^{10}$ for the photon assisted tunneling current through a superconducting tunnel junction. Note, however, that in the Tien-Gordon case the current has been considered as a function of bias voltage, whereas in our case, it is a function of $\epsilon_{0}$, the energy shift of the discrete levels. Analogously, the alternating field is not applied in the bias direction, but rather to the gate electrodes.

In Fig. 4, the current has been plotted as a function of $\epsilon_{0}$ and $\tilde{\boldsymbol{\epsilon}}$. The figure clearly shows that the current is composed of a number of satellite peaks each separated by the photon energy $\hbar \omega$. With increasing amplitude $\tilde{\epsilon}$, the number of visible current peaks increases. The peaks all have the same width $\Gamma_{R}$ and have heights given by $4 T^{2} J_{n}^{2}(\tilde{\epsilon} / \omega) / \Gamma_{R}$. In the limit of small amplitude, the height of the $n=1$ satellite peak reduces to $I_{\max }=T^{2} \tilde{\epsilon}^{2} / \Gamma_{R} \omega^{2}$, identical to our earlier result. Note that Eq. (21) for the current no longer contains $\Gamma_{L}$. Because the tunnel rate from lead to dot is much larger than the tunnel rate between the dots, the width of the level is, in this case, determined by $\Gamma_{R}$ and $T$ only.

In conclusion, we have presented a complete theoretical picture of the dc transport through a double quantum dot in the presence of external harmonic irradiation. The photoresponse of the system exhibits extra resonant peaks when the frequency of the external irradiation matches the energy difference between the discrete states. At a further increase of the irradiation intensity, this satellite peak becomes of the same order of magnitude as the main peak, but preserves the much smaller width. At small tunneling amplitudes and large irradiation amplitude extra satellite peaks appear in a pattern similar to that obtained for a Josephson junction by Tien and Gordon. ${ }^{10}$

We acknowledge fruitful discussions with L.P. Kouwenhoven, M. Büttiker, N.C. van der Vaart, and G.E.W. Bauer. This work is part of the research program of the "Stichting voor Fundamenteel Onderzoek der Materie" (FOM), which is financially supported by the "Nederlandse Organisatie voor Wetenschappelijk Onderzoek” (NWO).
${ }^{1}$ M. Büttiker and R. Landauer, Phys. Rev. Lett. 49, 1739 (1982).

${ }^{2}$ I. Bar-Joseph and S.A. Gurvitz, Phys. Rev. B. 44, 3332 (1991).

${ }^{3}$ N.S. Wingreen, A.P. Jauho, and Y. Meir, Phys. Rev. B 48, 8487 (1993).

${ }^{4}$ L.P. Kouwenhoven et al., Phys. Rev. Lett. 73, 3443 (1994).

${ }^{5}$ N.C. van der Vaart et al., Phys. Rev. Lett. 74, 4702 (1995).

${ }^{6}$ S.A. Gurvitz, Phys. Rev. B 44, 11924 (1991); the method used has been corrected in S.A. Gurvitz et al. (unpublished).
${ }^{7}$ A.V. Korotkov, D.V. Averin, and K.K. Likharev, Phys. Rev. B 49, 7548 (1994).

${ }^{8}$ Yu.V. Nazarov, Physica B 189, 57 (1993).

${ }^{9}$ The notation $|0\rangle$ does not imply that there are no electrons in the dots. It may denote any ground state $(N, M)$ with $N$ electrons in the left dot and $M$ in the right one. The resonance then occurs between the states $(N+1, M)(|a\rangle)$ and $(N, M+1)(|b\rangle)$.

${ }^{10}$ P.K. Tien and J.P. Gordon, Phys. Rev. 129, 647 (1963). 